Neu im ökom verlag

\title{
Jenseits tradierter Wege
}

\begin{abstract}
Viele Potenziale einer nachhaltigen Entwicklung sind nur durch regionale Kooperationen zu erschließen. Dem stehen jedoch einige Hürden entgegen. Wichtig ist, dass die verschiedenen Akteure in ihrer jeweiligen Sprache gezielt für diesen Prozess gewonnen werden. Als Beispiele für neve Umsetzungswege werden die Modernisierung der öffentlichen Verwaltungen, eine verbesserte Kommunikation dieser mit Unternehmen sowie die Kommunikation von Unternehmen und Jugendlichen in der Region diskutiert.
\end{abstract}

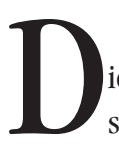
Von Petra Heuer und Angela Schilde ie beschleunigte ökonomische und gesellschaftliche Entwicklung führt zu neuen inhaltlichen und planerischen Herausforderungen. Dies erfordert problemorientierte, interdisziplinäre Ansätze in der Forschung sowie eine Verbesserung der Kommunikation und der Zusammenarbeit zwischen Verwaltung, Wissenschaft, EntscheidungsträgerInnen in Politik und Wirtschaft, Akteuren im Bildungs- und Ausbildungsbereich sowie der Öffentlichkeit.

Viele ökologische, soziale und ökonomische Probleme sind jedoch weder auf der nationalen noch auf der örtlichen Ebene lösbar. Nationale Programme und Pläne weisen für die konkrete Umsetzung einen zu generalisierenden Betrachtungsmaßstab auf. Die lokale Ebene erweist sich hierfür häufig als zu kleinräumig. Viele Potenziale einer nachhaltigen Entwicklung sind nur im regionalen Maßstab und durch regionale Kooperationen zu erschließen. Dies gilt auch im Hinblick auf die Einleitung einer Entwicklung hin zur Nachhaltigkeit (1). Als oberstes Ziel regionaler Entwicklung hat das Prinzip der Nachhaltigkeit in staatlichen Entwicklungsprogrammen Aufnahme gefunden. Daraus ergibt sich eine besondere Bedeutung von kommunalen und regionalen Netzwerken, die eine wichtige Rolle als Keimzelle für einen nachhaltigen Strukturwandel in der Region spielen können (2).

Die Diskussion einer nachhaltigen Entwicklung kann - um konkrete Ergebnisse liefern zu können und Handlungsalternativen aufzuzeigen - jedoch nur in einem breiten gesellschaftlichen Rahmen unter Einbezug des realen Lebensumfeldes der verschiedenen Akteure geführt werden. Um tragfähige Konzepte zu verwirklichen, muss ein breiter Konsens über die anzustrebenden Zielsetzungen sowie über die Wege der konkreten
Verwirklichung von Veränderungen erzielt werden. Hierzu ist eine Beteiligung der jeweiligen entscheidenden Akteure unerlässlich, denn nur diese setzen geplante Ideen und Konzepte anschließend in tatsächliches Handeln um.

\section{Hürden: Institutionalisierung ver- sus Flexibilität ...}

Innerhalb einer Kommune oder eines Kreises existieren zahlreiche formalisierte Kommunikationswege, zum Beispiel im Rahmen von Genehmigungsverfahren oder Meldepflichten. Diese werden jedoch vor allem mit Stichworten wie starrer Bürokratie und hohem Aufwand verbunden kaum jedoch mit Flexibilität und Mitgestaltung. Es gab verschiedene Versuche über die tradierten Kommunikationswege hinaus zu gelangen, etwa im Rahmen von Agenda-Prozessen. Grenzen werden vor allem dann deutlich, wenn tatsächlich nach Wegen der Einflussnahme und Beteiligung an Veränderungsprozessen gesucht wird sowie wenn das Geld aus Modellprojekten versiegt. Die Verstetigung solcher modellhaften Prozesse, die eine grundlegende Veränderung von Strukturen und Entscheidungswegen voraussetzen würde, ist bisher kaum gelungen.

Lösungsstrategien für eine nachhaltige Entwicklung sind jedoch untrennbar mit Fragen der Institutionalisierung und der Partizipation an Veränderungsprozessen verknüpft. Insbesondere bei Agenda-Prozessen wurde oftmals auf die Vorgabe bestimmter Strukturen und Richtungsanzeiger weitestgehend verzichtet, um die Prozesse nicht von vornherein in eine Form zu pressen. Mittlerweile aber hat sich genau dieses als Schwachstelle herausgestellt, sodass sie oftmals ins Leere laufen. Ein gangbarer Mittelweg zwischen Institutionalisierung und Flexibilität hat sich bisher nicht etabliert. Daher, aber auch weil die Akteure nicht

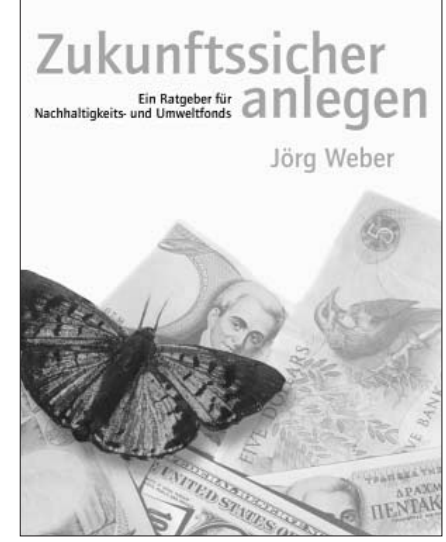

\section{Zukunftssicher anlegen}

Ein Ratgeber für Nachhaltigkeits- und Umweltfonds

Auf welche Energiequellen werden wir in zehn Jahren zurückgreifen? Wie werden wir uns in Zukunft ernähren? Wie werden wir reisen?

Die Antworten sind auch für das Thema Geldanlage wichtig. Zukunftssichere Fonds und Zertifikaten sollten nicht nur eine gute Rendite abwerfen, sondern auch das Umdenken in der Wirtschaft fördern. Jörg Weber porträtiert Umweltfonds- und Zertifikate sowie Fonds für erneuerbare Energien und Nachhaltigkeit. "Zukunftssicher anlegen" ermöglicht Renditevergleiche, aber auch Antworten auf Fragen wie: Welche Fonds legen in Wind- und Sonnenenergie-Aktien an? Welche investieren in Unternehmen, die keine Tierversuche durchführen?

Das Buch wendet sich an alle, die ihr Geld verantwortungsbewusst, zukunftssicher und rentabel anlegen wollen.

Jörg Weber, ökom Verlag 2001 184 Seiten, ISBN 3-928-24476-0

\section{www.oekom.de}

Für 15,00 € erhältlich beim ökom Verlag, Waltherstr. 29, 80337 München, Fon $++49 /(0) 89 / 54418$ 4-0, Fax $++49 /(0) 89 / 54418$ 4-49,

E-Mail kontakt@oekom.de,www.oekom.de 
mit genügend Handlungskompetenzen ausgestattet sind, bleibt eine Umsetzung in konkrete Aktionen und Maßnahmen häufig aus.

\section{>... und Tücken der Kommu- nikation über Nachhaltigkeit}

Schon unser ökologisches Handeln ist nicht primär von ökologischen Prinzipien geleitet. Unsere Alltagsentscheidungen sind in erster Linie von der gesellschaftlichen Umwelt und den Handlungsbedingungen abhängig. Die Menschen haben meistens keine Absicht die Umwelt zu gefährden, sondern sie müssen ihren alltäglichen Bedürfnissen nachgehen: zur Arbeit fahren, einkaufen, sich erholen, etc. Die Nebenfolgen dieser Aktivitäten sind Umweltbelastungen. Wenn das menschliche Handeln geändert werden soll, müssen also die realen Handlungsbedingungen mit berïcksichtigt werden (3). Dabei ist jedoch das Leitbild Nachhaltigkeit von ungleich größerer Komplexität und Abstraktion. Allein mit dem Begriff kann nur eine Minderheit der deutschen Bevölkerung überhaupt etwas anfangen. Zudem hat es einen hohen ideellen Wert. Das Interesse für ein ideelles Thema wird aber bei vielen Personen vor allem dann geweckt, wenn sie es mit etwas Praktischem oder Nützlichem verbinden können (4). Ein persönlicher Nutzen in Folge des eigenen Handelns ist jedoch bei einem Engagement in der Region häufig nicht direkt spürbar. Es besteht Ungleichheit und Ungleichzeitigkeit zwischen individuellem Nutzen und Gemeinnutz.

Will man die Diskussion zu Fragen einer nachhaltigen Entwicklung in allgemeine Lebens- und Lernzusammenhänge der Akteure integrieren gilt es, diese dort, also als Bürger, Mitarbeiter, Konsument etc., zu erreichen. Durch die Vernetzung der kommunalen und regionalen Akteure im Zuge gemeinsamer Aktivitäten hofft man, Synergieeffekte erzielen und nutzen zu können.

\section{Neve Wege ?!}

Wichtig ist, dass die verschiedenen Akteure in ihrer jeweiligen Sprache und mit ihren besonderen Interessen gezielt für diesen Prozess gewonnen werden und das Wirken ihres Handelns spüren. Dabei ist eine neue Kommunikationskultur notwendig, die sich durch alle Ebenen und Institutionen zieht.

Auf der Suche nach neuen Wegen scheint es sinnvoll, bereits bestehende Strukturen als Anknüpfungspunkt zu wählen, um diese dann für veränderte Fragestellungen flexibel und effektiv zu nutzen. Dabei bedarf es einer zielgruppenspe- zifischen ,An-Sprache“, um die wesentlichen Akteure zu aktivieren. An einzelnen Beispielen möchten wir aufzeigen, wie Möglichkeiten zur Umsetzung auf regionaler Ebene unserer Meinung nach aussehen könnten:

\section{Kommunikation in öffentlichen Verwaltungen}

Eine zukunftsfähige und wettbewerbsfähige Entwicklung auf lokaler und regionaler Ebene wird zunehmend auch von ökologischen und sozialen Gesichtspunkten bestimmt (Kundenzufriedenheit, Mitarbeiterorientierung etc.). Viele Untersuchungen zeigen, dass die Humanressourcen und ihr Management ein wichtiger Erfolgsfaktor sind, der den finanziellen Erfolg und die führende Stellung von Unternehmungen erklären kann (5). Verwaltungen sind insofern in ihrer Zusammensetzung als Dienstleistungsorganisation mindestens - wenn nicht weit mehr als Industrieunternehmen - vom Know-how ihrer Mitarbeitenden abhängig (6). Dauerhaft angestrebte Modernisierungen greifen jedoch nur, wenn sich das Selbstverständnis der Verwaltung und damit auch das Personal als Träger dieses Selbstverständnisses verändern (7).

Insofern gilt es, die abstrakte Nachhaltigkeitsdiskussion mittels Ziel-, Indikatoren- und Maßnahmenfestlegung zu konkretisieren, sodass die beziehungslose Thematik in konkrete Handlungsansätze für die Verwaltungsebene und ihre Beschäftigten umgesetzt wird. Zentrales Ziel ist es, die Verwaltungsbeschäftigten an den einzelnen Schritten zu beteiligen. Dies zum einen, um die tatsächlichen verwaltungsinternen Potenziale aufzugreifen und Probleme zu erkennen. Andererseits müssen die einzelnen Beschäftigten in ihrer täglichen Arbeit die bestehenden Leitideen und Zielsetzungen umsetzen. Um eine dauerhafte Institutionalisierung, aber auch eine Verselbständigung des Prozesses zu erreichen, gilt es vor Ort, also verwaltungsintern, „Trainer" in die Abläufe einzuweisen und in entsprechende Moderations- und Kommunikationsmethoden zu schulen. Ziel ist es, die Kommunikation innerhalb der Verwaltung anzuregen, aber auch eine Basis für eine breitere Nachhaltigkeitsdiskussion herzustellen.

\section{Kommunikation zwischen Verwaltungen und Unternehmen}

Das Verhältnis beispielsweise zwischen Kommunen und mittelständischen Unternehmen gestaltet sich nach wie vor schwierig. Eine Studie der Mittelstands-Offensive NRW hat insbesondere mangelhafte Kommunikation zwischen beiden Beteiligten als Hauptgrund für diese Problematik attestiert. Ursachen werden einerseits darin gesehen, dass die Kommunen die angewendeten Kommunikationswege und Instrumente zu wenig an den Bedürfnissen der klein- und mittelständischen Betriebe (KMU) ausrichten. So werden beispielsweise Bürgertelefone nur von wenigen Kommunen eingerichtet, bei KMU ist dieser Kommunikationsweg aber sehr beliebt. Aber auch die Unternehmen müssen mehr Eigeninitiative bei der Nutzung von Informations- und Serviceleistungen zeigen (8).

Im Zuge der sich ausweitenden lokalen und regionalen Agenda 21-Prozesse bilden sich andererseits immer mehr Initiativen heraus, in denen Gebietskörperschaften und Unternehmen versuchen, enger zusammen zu arbeiten und sich gegenseitig zu unterstïtzen. Konkrete Veränderungsprozesse sind jedoch noch nicht sehr weit gediehen. Themen wie Nachhaltigkeit, Ökoeffizienz und Ressourcenproduktivität sind für die meisten Unternehmen und Verwaltungen nach wie vor häufig noch Schlagwörter ohne konkrete Ansätze zur Realisierung. Dabei sprechen viele Fakten dafür, dass die Orientierung am Leitbild der Nachhaltigkeit Innovationspotenziale birgt, die gewinnbringend genutzt werden sollten (9). Ziel muss es deshalb sein, Kommunikationshürden zu überwinden und eine stärkere Zusammenarbeit beider Akteure auf kommunaler und regionaler Ebene zu unterstiutzen, um dieses häufig von kognitiven Dissonanzen geprägte Verhältnis zu entschärfen.

Positiv bewährt hat sich in diesem Zusammenhang die Initiative Umweltpakt Bayern: Hier gehen Zielvereinbarungen - und damit Mehraufwand für Unternehmen - und verbesserte Standortbedingungen durch staatliche Unterstiitzung Hand in Hand. Beispielsweise werden EMAS-registrierten Unternehmen von staatlicher Seite im Gegenzug verfahrensrechtliche Erleichterungen zugebilligt.

\section{Kommunikation regionaler Akteure - UmweltKURS}

2002 startet das auf vier Jahre ausgelegte und von der Deutschen Bundesstiftung Umwelt geförderte Projekt UmweltKURS in Kooperation zwischen dem Wuppertal Institut und dem Institut Unternehmen \& Schule. Im Rahmen dieses Projektes werden neue Möglichkeiten der Kommunikation in einer Region gesucht und erprobt. Ziel des Projektes ist es, einen Dialog zum Thema Nachhaltigkeit zwischen Jugendlichen und Unternehmen einer Region zu initiieren und langfristig zu institutionalisieren. Auch hier wird der Dialog zwischen den Akteuren, hier Produzenten und 
Konsumenten, als einer der wichtigsten Erfolgsfaktoren für eine nachhaltige Regionalentwicklung angesehen. Ziel ist eine Veränderung verfestigter Konsummuster und Lebensstile nicht nachhaltiger Art. Ein Ansatzpunkt hierfür kann neben dem „Appellieren an das Gute“ der Hinweis auf den eigenen Nutzen sein.

Welchen Nutzen bringt es Unternehmen und welchen Nutzen bringt es Schulen, sich daran zu beteiligen? Sowohl Bildungsinstitutionen als auch Unternehmen beklagen häufig eine tiefe Kluft zwischen Schule und Wirtschaft. Unternehmen sind an einer Kooperation mit Schulen interessiert, da sie motivierte und qualifizierte Arbeitskräfte suchen und eine zum Teil eklatante Realitätsferne von Schulen kritisieren. Mit dieser Kritik konfrontiert suchen Schulen zunehmend mehr Praxisbezug und versuchen, aktuelle Themen aufzugreifen. Insofern kann mit Lernpartnerschaften Schule - Wirtschaft an den Problemfeldern der Akteure angesetzt werden.

Das Eigeninteresse der beteiligten Akteure ist unserer Meinung nach eine notwendige aber nicht unbedingt ausreichende Voraussetzung, um solche Lernpartnerschaften langfristig zu institutionalisieren. Hierzu ist geplant, Schulbehörden und Wirtschaftsorganisationen wie die Industrieund Handelskammern als konkrete Träger vor Ort zu gewinnen und zentrale Büros zur Unterstïtzung der Prozesse zu verankern. Eine besondere Herausforderung des Projektes wird sein, Nachhaltigkeitsthemen - zum Beispiel zukunftsfähige Produktionsweisen und Konsummuster - so aufzubereiten, dass sie an die Lebenswelten der Beteiligten anschließen, Handlungsmöglichkeiten aufzeigen und in bestehende Lernzusammenhänge integriert werden können. Dabei gilt es die Sprache der Zielgruppe, hier also der Kinder und Jugendlichen, zu treffen.

\section{Nachhaltigkeit in die Breite tragen}

Die Nachhaltigkeitsdiskussion wurde bisher mit viel Idealismus geführt. Die regionalen Akteure als Zielgruppen mit unterschiedlicher Motivation für das eigene Handeln zu verstehen, wirkt vor diesem Hintergrund vielleicht zu nutzenorientiert. Soll die Debatte jedoch von ihrem akademischen Sockel in die breite Bevölkerung getragen werden und zu pragmatischem Handeln führen, so darf der Idealismus nicht allein im Vordergrund stehen. Die verschiedenen Sprachen und Interessen wesentlicher Akteure müssen stärker in den Mittelpunkt rücken. Allerdings bedarf es auch Mög- lichkeiten der Mitgestaltung, um Menschen zu motivieren, sich am Dialog über gesellschaftliche Veränderungsprozesse zu beteiligen. Deshalb ist es notwendig von der Kommunikation zu tatsächlicher Partizipation und verändertem Handeln zu gelangen. Der Dialog zwischen Konsumenten und Produzenten bietet hier genauso Anknüpfungspunkte wie die Modernisierung öffentlicher Verwaltungen und die Optimierung der Schnittstelle Unternehmen - Verwaltung.

\section{Anmerkungen}

(1) Behrendt, D. / Neitzke H.-P.: Kommunale und regionale Nachhaltigkeitsinventuren. ECOLOG-Bericht 1/2000.

(2) Rudolph, A.: Vernetzung regionaler Einflusseliten und ihr Beitrag zur Regionalentwicklung. In: Akademie für Raumforschung und Landesplanung: Zukunft von Räumen Räume mit Zukunft, Arbeitsmaterial Nr. 261, Hannover 1999, S. 12-18.

(3) Kyburz-Graber, R./ Rigendinger, L./ Hirsch, G./ Werner, K. : Lernen in der Umwelt: sozio-ökologische Umweltbildung. In: Roux, M./Bürgin, S. (Hrsg.): Förderung umweltbezogener Lernprozesse in Schulen, Unternehmen und Branchen, Birkhäuser 1996, S. 15-41; de Haan, G./ Kuckartz, U.: Phänomene des Umweltbewusstseins. In: Greenpeace (Hrsg.): Neue Wege in der Umweltbildung. Die Werkstatt 1995, S. 12-31.

(4) Bremer, H.: Alltagskulturen und Lernen bei Arbeitnehmerlnnen - Der Milieuansatz und seine Bedeutung für die Praxis. Vortrag zum Abschlussworkshop des Vorprojekts „Nachhaltige Entwicklung und Arbeitnehmerinteressen", 2001.

(5) Bolay, F.W.: Zum Konzept eines ressourcenorientierten Verwaltungsmanagement. In: Verwaltung und Management, 7. Jg. (2001), Heft 2, S. 100-108.

(6) Patig, J.: Kommunale Balanced Scorecard in der Praxis - Das Beispiel Horb am Neckar, in: IAW-Mitteilungen 4/2000, S. 17-23.

(7) B. Ruetz: Krise der öffentlichen Verwaltung. In: www. web-polis.de/hausarbeiten/lesen/archiv2.html

(8) Mittelstands-Offensive NRW: Kommunal- und Unternehmensbefragung der Mittelstands-Offensive NRW move, Düsseldorf 2001.

(9) Liedtke, C./ Baedeker, C./ Rohn, H./ Klemisch, H. (Hrsg.): Der Mittelstand gewinnt. Über Effizienz, Produkte und Allianzen. Stuttgart 2002.

\section{Die Autorinnen}

Petra Heuer und Angela Schilde sind wissenschaftliche Mitarbeiterinnen am Wuppertal Institut für Klima, Umwelt, Energie, AG „ökoeffizienz \& zukunftsfähige Unternehmen".

Kontakt: Wuppertal Institut, Döppersberg 19, 42103 Wuppertal. Tel. 0202/ 2492-239/-152, Fax-138, E-mail: petra.hever@wupperinst.org, angela.schilde@wupperinst.org

\section{Neuerscheinung: \\ UnternehmensGrün Band 8}

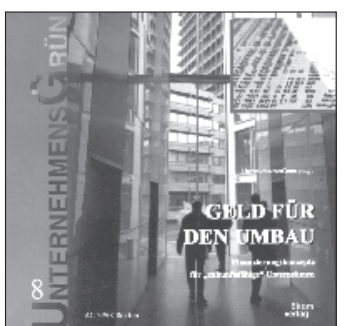

\section{Geld für den Umbau}

Finanzierungskonzepte für "zukunftsfähige" Unternehmen

Für kleine und mittlere Unternehmen wird es immer schwieriger, die notwendige Kapitalbasis für ihre sozialen und ökologischen Innovationen zu organisieren. Ihnen bleibt oft nur die mühevolle Suche nach Eigen- und Beteiligungskapital bei Investoren. UnternehmensGrün fasst daher entsprechende Finanzierungsmöglichkeiten zusammen. Autorlnnen aus Politik und Bankenwelt diskutieren die verschiedenen Methoden von Eigen- und Fremdkapitallösungen, über Mitarbeitermodelle und grüne Beteiligungsgesellschaften bis hin zur grünen Börse. "Geld für den Umbau" zeichnet die konkrete Vision einer alternativen Börse. Soziales und ökologisches Engagement führt langfristig - neben guten Renditen - zum "Fairholder value".

Unternehmensgrün, Verband zur Förderung umweltgerechten Wirtschaftens (Hrsg.), Band 8, 12,80€, ökom Verlag, ISBN 3-928244-73-6.

Band 1

"Augen zu und durch?

Was kommt nach der Krise?" - Über den konstruktiven Umgang mit der Rezession

Band 2

Erhalt durch Veränderung. Perspektiven einer ökologischen und sozialen Umsteuerung

Band 3

Konkurrenten oder Partner. Ökobilanz undf Öko-Audit im Verlgeich

Band 4

Von der Vision zur Praxis. Nachhaltiges Wirtschaften als Perspektive für Unternehmen

Band 5

Märkte ohne Grenzen.

Unternehmen im Spannungsfeld zwischen nachhaltigen Wirtschaften und globalen Wettbewerb

Band 6

Arbeit - Strategien der Existenzsicherung

Band 7

Mit neuer Energie in die Zukunft. Unternehmen zwischen Billigstrom- und Öko-Angeboten

Erhältlich bei pan adress,

Semmelweisstr. 8, D-82152 Planegg,

Fon $++49 /(0) 89 / 85709-145$, Fax -131,

E-Mail kontakt@oekom.de,www.oekom.de 
(c) 20I0 Authors; licensee IÖW and oekom verlag. This is an article distributed under the terms of the Creative Commons Attribution Non-Commercial No Derivates License (http://creativecommons.org/licenses/by-nc-nd/3.o/), which permits unrestricted use, distribution, and reproduction in any medium, provided the original work is properly cited. 